\section{Atendimento pré-hospitalar móvel em Fortaleza, Ceará: a visão dos profissionais envolvidos}

\author{
Mobile pre-hospital care in \\ Fortaleza, Ceará: the vision of \\ professionals involved
}

\author{
Juliana Guimarães e Silva' \\ Luiza Jane Eyre de Souza Vieira' \\ Augediva Maria Jucá Pordeus',"I \\ Edinilsa Ramos de Souza"' \\ Marcelo Luiz Carvalho Gonçalves' \\ 'Centro de Ciências da Saúde. Mestrado em Saúde Coletiva. Universidade de \\ Fortaleza. \\ "Núcleo de Epidemiologia da Secretaria de Saúde do Estado do Ceará - SESA. \\ II Centro Latino-Americano de Estudos de Violência e Saúde Jorge Careli. Escola \\ Nacional de Saúde Pública Sérgio Arouca. Fundação Oswaldo Cruz, Rio de \\ Janeiro, RJ.
}

\section{Resumo}

Com o objetivo de descrever variáveis sociodemográficas, profissionais e operacionais das diferentes categorias envolvidas no Serviço de Atendimento Móvel de Urgência, bem como a percepção destas quanto à adequação do serviço oferecido às diretrizes da Política Nacional de Atenção às Urgências, foi realizado um estudo transversal, mediante um questionário autoaplicável, em 2007/2008. Os 89 pesquisados apontaram comprometimento da estrutura física (60,7\%); escassez de materiais $(82,0 \%)$; incipiência de recursos humanos $(37,1 \%)$; mau estado de conservação e número insuficiente de ambulâncias (67,4\%). 98,9\% admitiram que há integração com outros serviços, com diferença estatística entre as categorias profissionais ( $\mathrm{p}=0,037)$. A terceirização predominou entre $71,4 \%$ dos médicos e $84,2 \%$ dos enfermeiros ( $\mathrm{p}<$ $0,001)$. A capacitação específica foi confirmada por $79,8 \%$ e a atualização por $88,8 \%$ dos profissionais. A média de tempo de resposta total foi de 29 minutos ( $\mathrm{dp} \pm 14,8$ ) e $65,2 \%$ afirmaram conhecer a política. Este estudo evidenciou que o pré-hospitalar é um serviço com problemas estruturais e de planejamento, nos quais se destacaram a precariedade das condições de trabalho e a fragilidade dos vínculos.

Palavras-chave: Assistência Pré-Hospitalar. Serviços Pré-Hospitalares. Emergências. Políticas Públicas de Saúde. 


\section{Abstract}

A cross-sectional study based on a self-administered questionnaire was performed in $2007 / 2008$, with the objective of describing sociodemographic, professional, and operating variables of the various professional categories involved in the Mobile Emergency Care Service, and the perception of the compliance of the service offered with the guidelines of the National Emergency Care Policy. All 89 participants indicated deficient physical structure $(60.7 \%)$; shortage of materials $(82.0 \%)$; unskilled human resources (37.1\%); poor conservation conditions and insufficient number of ambulances (67.4\%); 98.9\% admitted the existence of integration with other services, with some statistical differences among professional categories ( $\mathrm{p}=0.037$ ). Outsourcing prevailed among $71.4 \%$ of physicians and $84.2 \%$ of nurses ( $p<0.001$ ). Specific capacity building was confirmed by $79.8 \%$, and update by $88.8 \%$ of professionals. Total average response time was 29 minutes ( $\mathrm{SD} \pm 14.8$ ), and $65.2 \%$ acknowledged knowing the policy. This study showed that pre-hospital care services have structural and planning problems, among which poor working conditions and fragile ties stand out.

Keywords: Pre-hospital Care. Pre-hospital Care Services. Emergencies. Public Health Policies.

\section{Introdução}

As modificações no perfil epidemiológico decorrentes do crescimento das causas externas sobrecarregam os serviços de urgência e emergência, ocasionando intenso impacto sobre o sistema de saúde. Essa situação é agravada em muitos casos, porque esses serviços ainda atendem pessoas que não se enquadram na complexidade desse cuidado, como ressaltado pela Portaria $2.048^{1}$.

Diversos aspectos precedem a porta do hospital, onde essa demanda, associada às emergências clínicas, é atendida pelos serviços de atendimento pré-hospitalar móvel. Esta assistência ocorre em vias públicas, no domicílio ou em locais públicos, por meio da utilização de recursos adequados à complexidade do caso ${ }^{2,3}$.

No âmbito do SUS, o atendimento préhospitalar (APH) se divide em dois tipos de serviços: pré-hospitalar fixo e móvel. O pré-hospitalar móvel, foco deste estudo, é constituído pelo Serviço de Atendimento Móvel de Urgência - SAMU e serviços associados de salvamento e resgate ${ }^{4}$.

O socorro móvel é solicitado gratuitamente pelo telefone 192. A ligação é atendida por técnicos da central de regulação, que identificam a emergência e a transferem para o médico regulador. Este profissional faz o diagnóstico da situação e classifica a urgência, definindo o recurso necessário ao atendimento. Este varia desde uma orientação ao paciente ou ao indivíduo que realizou a chamada, até o envio ao local da ocorrência de uma unidade de Suporte Básico ou Avançado de Vida ${ }^{5}$.

Não se pode negar a contribuição do APH para a redução do tempo de chegada ao hospital adequado e para o sucesso das intervenções iniciais apropriadas à manutenção da vida ${ }^{6}$. A literatura publicada nos anos 80, nos Estados Unidos e em países da Comunidade Européia, mostra que a percentagem de mortes evitáveis decresceu $50 \%$ após a implantação desses serviços ${ }^{7}$.

Durante a década de 80 , a ausência de diretrizes nacionais para a área de emergência, em especial para o APH, levou alguns 
estados a criar seus próprios serviços, sem normalização, planejamento e operacionalização, o que resultou em deficiências técnicas importantes nesse atendimento ${ }^{7}$. Em 2003, foi instituída a Política Nacional de Atenção às Urgências - PNAU, que surgiu com base nos dados demonstrados na Política de Redução da Morbimortalidade por Acidentes e Violências. Devendo ser implantada em todas as unidades federadas, a PNAU estabelece normas para a organização dos serviços públicos e privados nas esferas estadual, regional e municipal e define os seus componentes, entre eles, o pré-hospitalar móvel ${ }^{4,7}$.

Neste contexto, o SAMU é uma estratégia para a implantação da PNAU, que segue os princípios do Sistema Único de Saúde e combina ações de prevenção, atenção individual e coletiva. Resultou da união de áreas técnicas diversas que buscaram definir protocolos, fluxos de referência e contrarreferência, articulando os serviços, municípios e Estados, buscando uma atenção humanizada e integral ${ }^{8}$.

A rede nacional SAMU possui 147 serviços no Brasil. São atendidos 1.273 municípios, totalizando 112.546.443 milhões de pessoas, estando presente em 26 capitais brasileiras ${ }^{9}$. O SAMU de Fortaleza foi implantado em 2003 e, atualmente, é gerenciado pela Secretaria Municipal de Saúde, realizando, em média, seis mil atendimentos por mês (SMS, 2008). Investigar essa temática é importante em virtude da complexidade do APH Móvel e da operacionalização das diretrizes da PNAU, contribuindo com a produção do conhecimento na consolidação das políticas do SUS.

Parte de um estudo maior, que mapeou o atendimento pré-hospitalar móvel sob a ótica dos profissionais do serviço em Fortaleza, Ceará, este artigo teve por objetivos descrever variáveis sociodemográficas e profissionais das diferentes categorias envolvidas no SAMU, além de descrever variáveis operacionais do serviço. Analisou, ainda, a percepção dessas categorias quanto à adequação do serviço oferecido às diretrizes da PNAU.

\section{Metodologia}

Trata-se de um estudo transversal, descritivo, tendo como unidade de análise os profissionais das equipes do Serviço de Atendimento Móvel de Urgência, em Fortaleza, Ceará, em 2007/2008. O estudo foi desenvolvido nos 11 pontos de apoio e na central do SAMU, cuja frota era constituída em sua totalidade por 21 veículos, sendo 17 de Suporte Básico de Vida e 4 de Suporte Avançado de Vida. As ambulâncias, com suas referidas equipes, eram distribuídas em 11 bairros de Fortaleza.

Foram convidados a participar do estudo todos os 260 profissionais das equipes de atendimento do SAMU no Município, independentemente do vínculo empregatício. Estes profissionais eram divididos em 5 equipes, que se revezavam na escala de plantão (12h) diurno e noturno, integrando as equipes de Suporte Básico e Avançado de Vida. O universo de estudo englobou médicos, enfermeiros, técnicos e auxiliares de enfermagem, bem como os condutores de veículos de urgência.

O instrumento de coleta de dados, pré-testado e autoaplicável, abordou as seguintes variáveis:

- variáveis sociodemográficas e profissionais (sexo, idade, tempo de trabalho no SAMU, função desempenhada, trabalho em outro serviço pré-hospitalar e curso de aperfeiçoamento em atendimento pré-hospitalar);

- indicadores de estrutura e organização do SAMU, tendo como referência a PNAU (estrutura física, recursos materiais, recursos humanos, ambulâncias, integração com outros serviços e atualização profissional propostas pelo serviço);

- tempo médio de resposta total, estimado pelos profissionais;

- existência de referência e contrarreferência;

- conhecimento do profissional acerca da PNAU; e

- capacitação realizada por essas equipes. 
A entrega dos questionários obedeceu aos seguintes passos:

- explicação do objetivo da pesquisa aos profissionais dos pontos de apoio;

- entrega dos questionários ao profissional de plantão;

- orientação ao profissional, para que este passasse o questionário à equipe do plantão seguinte;

- recolhimento dos questionários devolvidos nos pontos de apoio, após o período 60 dias.

Por motivos operacionais, tais como dificuldade de acesso e exposição a áreas de risco indicadas por um gestor do SAMU, não foi possível a entrega individual do questionário a todos os profissionais envolvidos.

Os dados foram analisados pelo programa Statistical Package for the Social Sciences - SPSS, versão 13.0. Foram feitas análises univariadas e bivariadas. Para se verificar associação entre as variáveis, foi utilizado o teste Qui-Quadrado de Pearson, considerando-se $\mathrm{p} \leq 0,05$, num grau de confiabilidade de $95 \%$.

Foi respeitada a Resolução no 196/96, do Ministério da Saúde (Conselho Nacional de Saúde $)^{10}$. Os participantes foram informados acerca dos objetivos da pesquisa em reunião efetuada antes do seu início e, além disso, assinaram um termo de consentimento livre e esclarecido, que explicitou o objetivo principal da pesquisa e assegurou o sigilo, o anonimato, a confidencionalidade, a privacidade e o direito de excluir-se desta. O estudo foi submetido ao Comitê de Ética e Pesquisa da Universidade de Fortaleza e aprovado mediante o Parecer nº306/2007.

\section{Resultados}

Dos 260 questionários distribuídos, foram devolvidos 89 (34,2\%). Considerandose apenas os 89 que devolveram o questionário, $61,8 \%$ pertenciam ao sexo masculino e $38,2 \%$ ao feminino, tendo a maior parte deles (54,0\%) idade compreendida entre 31 e 40 anos, com média de 36,2 anos e desviopadrão de $\pm 11,2$. Desses profissionais, 7,9\% eram médicos, $21,3 \%$ enfermeiros, $33,7 \%$ técnicos e auxiliares de enfermagem e 37,1\% condutores de veículos de urgência.

O tempo de trabalho na instituição predominou entre 1 e 5 anos (43,8\%). A média para o tempo de trabalho no SAMU foi de 7,6 anos, com desvio-padrão de $\pm 4,9$. Dos profissionais pesquisados, $51,7 \%$ relataram que não trabalhavam ou trabalharam em outro serviço de APH. Dentre os que afirmaram exercer o APH em outra instituição, $58,1 \%$ o faziam por um período entre 1 e 5 anos, coincidindo com o tempo de trabalho no SAMU. A média calculada para o tempo de trabalho em outro serviço pré-hospitalar correspondeu a 6,0 anos, com desviopadrão de $\pm 5,3$ (Tabela 1).

Ao analisar o funcionamento do serviço, os profissionais $(60,7 \%)$ relataram que a estrutura física do SAMU deixa a desejar, porém atende a demanda. Isto foi identificado por $71,4 \%$ dos médicos e $70,0 \%$ dos técnicos e auxiliares de enfermagem. A escassez de recursos materiais foi apontada por $82,0 \%$ dos profissionais. Neste contexto, $90,7 \%$ dos condutores, $80 \%$ dos técnicos e auxiliares, $73,7 \%$ dos enfermeiros e $71,4 \%$ dos médicos apontaram que às vezes falta material para o atendimento. $37,1 \%$ deles informaram que a instituição possui recursos humanos em número insuficiente. Isso, porém, não compromete o atendimento. Os médicos $(57,1 \%)$ foram a categoria profissional que mais identificou essa deficiência. Quanto ao estado de conservação dos veículos de urgência (ambulâncias), 67,4\% da equipe referiu que essas se apresentam em estado comprometido e em número insuficiente para o adequado atendimento sendo mais comumente identificados pelos condutores de veículos de urgência $(78,2 \%)$ e pelos médicos $(71,4 \%)$.

No período deste estudo, a frota do SAMU Fortaleza possuía somente ambulâncias do tipo B e D (Suporte Básico - 17 veículos; e Avançado de Vida - 4 veículos) e veículos leves, denominados carros médicos.

A integração com outros serviços foi confirmada por $98,9 \%$ dos profissionais e ocorre principalmente com o Corpo de 
Tabela 1 - Características sociodemográficas da equipe do Serviço de Atendimento Móvel de Urgência (SAMU). Fortaleza, 2008.

Table 1 - Sociodemographic characteristics of the staff of Mobile Emergency Care Service (SAMU). Fortaleza, 2008.

\begin{tabular}{|c|c|c|}
\hline Variáveis sociodemográficas & $\mathrm{N}$ & $\%$ \\
\hline \multicolumn{3}{|l|}{ Sexo } \\
\hline Masculino & 55 & 61,8 \\
\hline Feminino & 34 & 38,2 \\
\hline \multicolumn{3}{|l|}{ Idade } \\
\hline 21 a 30 & 11 & 12,3 \\
\hline 31 a 40 & 48 & 54,0 \\
\hline 41 a 50 & 21 & 23,6 \\
\hline 51 a 60 & 04 & 4,5 \\
\hline Não respondeu & 05 & 5,6 \\
\hline \multicolumn{3}{|l|}{ Categoria Profissional } \\
\hline Médico & 07 & 7,9 \\
\hline Enfermeiro & 19 & 21,3 \\
\hline Técnico/Auxiliar de Enfermagem & 30 & 33,7 \\
\hline Condutor de Veículo de Urgência & 33 & 37,1 \\
\hline \multicolumn{3}{|l|}{ Tempo de trabalho no SAMU (anos) ${ }^{1}$} \\
\hline 1 a 5 & 39 & 43,8 \\
\hline 6 a 10 & 21 & 23,6 \\
\hline 11 a 15 & 27 & 30,4 \\
\hline 16 a 20 & 02 & 2,2 \\
\hline \multicolumn{3}{|c|}{ Trabalha ou trabalhou em outro SAPH ${ }^{2}$} \\
\hline Não & 46 & 51,7 \\
\hline Sim & 43 & 48,3 \\
\hline \multicolumn{3}{|c|}{ Tempo de Trabalho em Outro SAPH (anos) } \\
\hline 1 a 5 & 25 & 58,1 \\
\hline 6 a 10 & 13 & 30,2 \\
\hline 11 a 15 & 01 & 2,4 \\
\hline Não respondeu & 04 & 9,3 \\
\hline Total & 89 & 100 \\
\hline
\end{tabular}

'Tempo de trabalho em outro serviço de APH: porcentagens calculadas com base no número de profissionais que afirmaram trabalhar ou terem trabalhado em outro serviço de APH./ Time working at another prehospital care service: percentages calculated based on the number of professionals that work or have worked in another prehospital care department.

${ }^{2} \mathrm{SAPH}$ - Serviço de atendimento pré-hospitalar./Prehospital care.

Bombeiros (85,6\%), Polícia Militar (76,4\%), Polícia Rodoviária $(74,1 \%)$, agentes de trânsito $(47,1 \%)$ e Polícia Civil $(26,7 \%)$. Na análise do funcionamento do SAMU pelas diferentes categorias profissionais, houve diferença estatisticamente significativa entre a categoria profissional e a integração com outros serviços $(p=0,037)$. Os médicos interagem menos com os outros serviços do que os demais profissionais (Tabela 2).

Quanto ao vínculo empregatício, 65,2\% eram servidores públicos e $33,7 \%$ terceirizados. De acordo com a categoria profissional, predominaram os servidores públicos entre os profissionais técnicos: condutores de veículos de urgência $(87,5 \%)$ e técnicos/ 
Tabela 2 - Distribuição percentual dos profissionais do Serviço de Atendimento Móvel de Urgência para variáveis relacionadas ao atendimento do SAMU, de acordo com a categoria profissional. Fortaleza, 2008.

Table 2 - Percentage distribution of Mobile Emergency Care Service for variables related to the SAMU, according to professional category. Fortaleza, 2008.

\begin{tabular}{|c|c|c|c|c|c|}
\hline \multirow[t]{2}{*}{ Variáveis de Funcionamento } & \multicolumn{5}{|c|}{ Categoria Profissional $(\mathrm{N}=89)$} \\
\hline & $\begin{array}{l}\text { Médico } \\
(\mathrm{n}=07)\end{array}$ & $\begin{array}{l}\text { Enfermeiro } \\
\quad(\mathrm{n}=19)\end{array}$ & $\begin{array}{l}\text { Técnico/Auxiliar de } \\
\text { Enfermagem } \\
(n=30)\end{array}$ & $\begin{array}{l}\text { Condutor } \\
(\mathrm{n}=33)\end{array}$ & Valor de $\mathrm{p}^{*}$ \\
\hline \multicolumn{6}{|l|}{ Estrutura física } \\
\hline Bem estruturado, atende à demanda & --- & --- & --- & 3,1 & 0,856 \\
\hline $\begin{array}{l}\text { Atende a demanda, pode melhorar a } \\
\text { estrutura. }\end{array}$ & 14,3 & 47,4 & 23,3 & 18,8 & \\
\hline $\begin{array}{l}\text { A estrutura deixa a desejar mas atende a } \\
\text { demanda. }\end{array}$ & 71,4 & 42,1 & 70,0 & 62,5 & \\
\hline $\begin{array}{l}\text { Não possui estrutura para atender a } \\
\text { demanda }\end{array}$ & 14,3 & 10,5 & 6,7 & 15,6 & \\
\hline Não respondeu & --- & --- & --- & --- & \\
\hline \multicolumn{6}{|l|}{ Recursos materiais } \\
\hline Há sempre material disponível & 14,3 & 26,3 & 6,7 & 3,1 & 0,620 \\
\hline As vezes falta material. & 71,4 & 73,7 & 80,0 & 90,7 & \\
\hline Não há material disponível & 14,3 & --- & 13,3 & 6,2 & \\
\hline Não respondeu & --- & --- & --- & --- & \\
\hline \multicolumn{6}{|l|}{ Recursos humanos } \\
\hline Suficiente ao bom funcionamento do SAMU & 14,3 & 26,3 & 36,7 & 25,0 & 0,809 \\
\hline $\begin{array}{l}\text { Insuficiente, porém não compromete o } \\
\text { funcionamento }\end{array}$ & 28,6 & 31,6 & 40,0 & 28,1 & \\
\hline $\begin{array}{l}\text { Insuficiente e compromete o } \\
\text { funcionamento }\end{array}$ & 57,1 & 36,9 & 23,3 & 43,8 & \\
\hline Não respondeu & --- & 5,2 & --- & 3,1 & \\
\hline \multicolumn{6}{|l|}{ Ambulâncias } \\
\hline $\begin{array}{l}\text { Bom estado de conservação, número } \\
\text { suficiente. }\end{array}$ & --- & 5,2 & --- & 3,1 & 0,474 \\
\hline $\begin{array}{l}\text { Bom estado de conservação, número } \\
\text { insuficiente }\end{array}$ & 28,6 & 26,3 & 13,3 & 3,1 & \\
\hline $\begin{array}{l}\text { Estado de conservação comprometido, } \\
\text { número suficiente }\end{array}$ & --- & 10,5 & 26,7 & 15,6 & \\
\hline $\begin{array}{l}\text { Estado de conservação comprometido, } \\
\text { número insuficiente }\end{array}$ & 71,4 & 58,0 & 60,0 & 78,2 & \\
\hline Não respondeu & --- & --- & --- & --- & \\
\hline \multicolumn{6}{|l|}{ Integração com outros serviços } \\
\hline $\operatorname{Sim}$ & 85,7 & 100,0 & 100,0 & 100,0 & 0,037 \\
\hline Não & 14,3 & --- & --- & --- & \\
\hline Não respondeu & --- & --- & -- & --- & \\
\hline
\end{tabular}

*Teste do qui-quadrado de Pearson; significativo quando $p \leq 0,05 . /{ }^{*}$ The Pearson's Chi-Square Test, significant at $p \leq 0.05$.

auxiliares de enfermagem $(83,3 \%)$. Os profissionais de nível superior são, em sua maioria, terceirizados: enfermeiros $(84,2 \%)$ e médicos $(71,4 \%)$. Essas diferenças mostraram-se estatisticamente significativas $(\mathrm{p}<0,001)$.
Neste estudo foram considerados como capacitação específica os cursos destinados ao treinamento dos profissionais de saúde para o desenvolvimento do atendimento pré-hospitalar. Desse modo, 79,8\% dos profissionais asseguraram que a realizaram: 
$75,3 \%$ por meio de cursos de atendimento básico de vida, $11,2 \%$ se capacitaram em suporte avançado de vida, 2,2\% buscaram a pós-graduação e 21,3\% deles não especificaram o curso realizado. A categoria profissional que mais procurou cursos na área foram os técnicos/auxiliares de enfermagem (93,3\%). Entre os profissionais de nível superior, os enfermeiros foram os que mais buscaram se preparar para o exercício do APH (68,4\%). A atualização profissional oferecida pelo SAMU foi relatada por $88,8 \%$ dos profissionais. As principais atividades citadas para este fim foram: cursos de curta duração $(40,4 \%)$, palestras $(34,8 \%)$, cursos de 40 horas ou mais $(25,8 \%)$, educação continuada $(23,5 \%)$ e educação em serviço (7,8\%).

O tempo de resposta total relatado pelos profissionais foi de 31 a 40 minutos (30,3\%), correspondendo à média de 29,0 com desvio-padrão de $\pm 14,8$. Na análise do conceito de tempo médio de resposta total, observaram-se respostas inadequadas emitidas por $48,3 \%$ dos servidores. Efetuandose a análise por categoria profissional, os médicos $(57,1 \%)$ e os técnico/auxiliares de enfermagem $(56,7 \%)$ foram os que mais responderam adequadamente sobre este conceito preconizado pela PNAU. Os enfermeiros $(52,5 \%)$ foram os que mais erraram essa definição.

Os problemas no sistema de referência e contrarreferência foram apontados por 95,5\% da equipe de saúde do SAMU, sendo mencionados por $100,0 \%$ dos médicos. Estes se traduziram por falta de vagas nos hospitais (53,9\%), escassez de equipamentos $(48,3 \%)$, má vontade dos profissionais da rede hospitalar em receber os pacientes atendidos pela equipe do SAMU $(40,4 \%)$, carência de especialistas para assistir adequadamente os pacientes $(28,0 \%)$, falhas na integração entre o SAMU e a rede de atenção hospitalar $(26,9 \%)$. Os profissionais, igualmente, certificaram $(75,3 \%)$ que existem rotinas e protocolos de atendimento e $60,6 \%$ garantiram que esses são cumpridos no cotidiano do trabalho. Os enfermeiros predominaram nestas afirmações, com 79,0\% e $73,7 \%$, respectivamente. No tocante ao conhecimento referido pelas equipes acerca da PNAU, 65,2\% relataram que conhecem a política e $42,7 \%$ confirmaram que o serviço não funciona em consonância com o preconizado por esse documento (Tabela 3 ).

\section{Discussão}

A análise do perfil dos profissionais do Serviço de Atendimento Móvel de Urgência de Fortaleza assemelha-se a dados encontrados em estudo acerca do APH móvel, realizado em Ribeirão Preto, no qual foram identificados a predominância do sexo masculino (67,5\%), idade entre 28 e 48 anos (95,0\%), tempo de exercício na instituição inferior a cinco anos (65,0\%) e experiência em outro serviço pré-hospitalar por período de 1 a 5 anos (62,5\%). Em relação à categoria profissional, pesquisa similar realizada em Belo Horizonte demonstrou que os técnicos/auxiliares de enfermagem $(47,5 \%)$ e condutores de veículos de urgência $(27,7 \%)$ prevalecem nos estudos ${ }^{11,12}$.

A predominância do sexo masculino na faixa etária jovem pode ser atribuída ao esforço físico que esses profissionais realizam ao desenvolver as atividades cotidianas, a exemplo da movimentação de macas e peso excessivo. Quanto ao tempo de trabalho no SAMU inferior a 5 anos, este fato pode ser explicado pela criação do serviço em 2004 e pode estar interligado ao fato de o vínculo empregatício desses com a instituição ser caracterizado pela terceirização.

A incipiência da estrutura física, escassez de recursos materiais, humanos e ambulâncias, e falhas na integração com outros serviços, apontaram deficiências que comprometem o desempenho e a qualidade da assistência. Estes problemas remetem diretamente à gestão dos recursos do sistema e regulamentação do componente pré-hospitalar móvel pela PNAU. Neste sentido, os atendimentos nas emergências demandam procedimentos e tecnologias específicas prontamente disponíveis ${ }^{13}$.

A Portaria $n^{0} 2.048 / G M$ preconiza os equipamentos e medicamentos para que 
Tabela 3 - Distribuição percentual dos profissionais do Serviço de Atendimento Móvel de Urgência para variáveis relacionadas ao trabalho, conhecimento da Política Nacional de Atenção às Urgências segundo a categoria profissional. Fortaleza, 2008.

Table 3 - Distribution of the professionals of the Mobile Emergency Care Service for variables related to work, knowledge of the National Emergency Care Policy according to professional category. Fortaleza, 2008.

\begin{tabular}{|c|c|c|c|c|c|}
\hline \multirow[t]{2}{*}{ Variáveis } & \multicolumn{5}{|c|}{ Categoria Profissional $(\mathrm{N}=89)$} \\
\hline & $\begin{array}{l}\text { Médico } \\
(\mathrm{n}=07)\end{array}$ & $\begin{array}{l}\text { Enfermeiro } \\
(\mathrm{n}=19)\end{array}$ & $\begin{array}{l}\text { Técnico/Auxiliar de } \\
\text { Enfermagem } \\
(n=30)\end{array}$ & $\begin{array}{l}\text { Condutor } \\
(n=33)\end{array}$ & Valor de $\mathrm{p}^{*}$ \\
\hline \multicolumn{6}{|l|}{ Vínculo } \\
\hline Servidor público & 28,6 & 15,8 & 83,3 & 87,5 & $<0,001$ \\
\hline Terceirizado & 71,4 & 84,2 & 16,7 & 12,5 & \\
\hline Não respondeu & --- & --- & --- & --- & \\
\hline \multicolumn{6}{|c|}{ Capacitação específica em APH } \\
\hline Sim & 42,9 & 68,4 & 93,3 & 81,3 & 0,074 \\
\hline Não & 57,1 & 31,6 & 3,3 & 6,2 & \\
\hline Não respondeu & --- & --- & 3,3 & 12,5 & \\
\hline \multicolumn{6}{|c|}{ Atualização Profissional } \\
\hline Sim & 85,7 & 89,5 & 86,7 & 90,7 & 0,699 \\
\hline Não & 14,3 & --- & 6,7 & 6,2 & \\
\hline Não respondeu & --- & 10,5 & 6,6 & 3,1 & \\
\hline \multicolumn{6}{|c|}{ Conceito de Tempo Resposta } \\
\hline Incorreto & 42,9 & 52,5 & 43,3 & 53,1 & 0,768 \\
\hline Correto & 57,1 & 37,0 & 56,7 & 40,7 & \\
\hline Não respondeu & --- & 10,5 & --- & 6,2 & \\
\hline \multicolumn{6}{|c|}{$\begin{array}{l}\text { Problemas no sistema de referência e } \\
\text { contrarreferência }\end{array}$} \\
\hline Sim & 100,0 & 94,8 & 96,7 & 93,8 & 0,763 \\
\hline Não & --- & --- & --- & 3,1 & \\
\hline Não respondeu & --- & 5,2 & 3,3 & 3,1 & \\
\hline \multicolumn{6}{|c|}{$\begin{array}{l}\text { Existência de rotinas e protocolos de } \\
\text { atendimento às vítimas de trânsito }\end{array}$} \\
\hline Sim & 71,6 & 79,0 & 73,3 & 75,0 & 0,950 \\
\hline Não & 14,2 & 10,5 & 6,7 & 12,4 & \\
\hline Não respondeu & 14,2 & 10,5 & 20,0 & 12,5 & \\
\hline \multicolumn{6}{|c|}{ Cumprimento de rotinas e protocolos } \\
\hline $\operatorname{Sim}$ & 57,4 & 73,7 & 73,3 & 56,3 & 0,380 \\
\hline Não & 28,4 & 15,8 & 10,0 & 15,6 & \\
\hline Não respondeu & 14,2 & 10,5 & 16,6 & 28,1 & \\
\hline \multicolumn{6}{|c|}{ Conhecimento da PNAU 1} \\
\hline Sim & 100,0 & 73,8 & 66,7 & 53,1 & 0,214 \\
\hline Não & --- & 21,0 & 30,0 & 40,7 & \\
\hline Não respondeu & & 5,2 & 3,3 & 6,2 & \\
\hline \multicolumn{6}{|c|}{$\begin{array}{l}\text { Funcionamento do SAMU em consonância } \\
\text { com a PNAU }\end{array}$} \\
\hline Sim & 28,6 & 31,6 & 23,3 & 12,5 & 0,518 \\
\hline Não & 57,1 & 63,2 & 73,3 & 59,3 & \\
\hline Não respondeu & 14,3 & 5,2 & 3,3 & 28,2 & \\
\hline
\end{tabular}


o APH seja efetuado com qualidade ${ }^{1}$, evitando-se assim a carência de recursos materiais. Pesquisa multicêntrica realizada em cinco capitais brasileiras - Recife, Manaus, Brasília, Rio de Janeiro e Curitiba - acerca da implementação do sistema de APH móvel, mostrou que apenas o Rio de Janeiro atende aos critérios de provisão de materiais. Brasília e Manaus foram os que exibiram mais limitações ${ }^{14}$. Estabelecendo um paralelo entre os resultados do estudo de Minayo e Deslandes ${ }^{14}$ com os achados deste estudo, percebe-se que a escassez dos recursos materiais não se distancia das deficiências encontradas em Brasília e Manaus, o que denota a incompatibilidade entre o discurso oficial da Política Nacional de Atenção às Urgências e o funcionamento do SAMU. Já a carência de recursos humanos assemelha-se às situações encontradas em Brasília, onde o gestor do SAMU avaliou com nota 6 , em uma escala de 0 a 10 , o número de profissionais disponíveis para $\mathrm{o}$ atendimento ${ }^{14}$.

Da mesma forma, os veículos apropriados ao atendimento pré-hospitalar são definidos em consonância com a PNAU. Esta determina que as ambulâncias são veículos terrestres, aéreos ou aquaviários destinados ao transporte exclusivo de pacientes e se classificam em: Tipo A - próprias para as remoções simples de caráter eletivo; Tipo B - adequadas ao Suporte Básico de Vida para paciente com risco de morte em transporte inter-hospitalar e paciente do APH com risco de morte desconhecido; Tipo C - destina-se ao resgate, atendimento pré-hospitalar de vítimas de acidentes ou que estejam em locais de acesso difícil e possuem equipamento de salvamento; Tipo D - objetiva o Suporte Avançado de Vida do paciente de alto risco e de transporte interhospitalar para os que precisam de cuidados médicos intensivos e uso de equipamentos; Tipo E - refere-se à aeronave de asa fixa ou rotativa para o resgate do paciente e transporte inter-hospitalar; Tipo F - embarcação para transporte em via marítima ou fluvial. Além dessas modalidades, há os meios de intervenção rápida, que utilizam veículos leves para o transporte de médicos e equipamentos, com vistas a realizar o suporte avançado de vida ${ }^{4}$.

A Política Nacional de Atenção às Urgências preconiza que as ambulâncias devem ser adquiridas na proporção de um veículo de Suporte Básico de Vida (SBV) para cada 100.000 a 150.000 habitantes, e de um veículo de Suporte Avançado de Vida (SAV) para cada 400.000 a 450.000 habitantes ${ }^{15}$. Fortaleza possui atualmente, de acordo com o IBGE ${ }^{16}, 2.431 .415$ habitantes. À vista desta informação, o número de ambulâncias de SBV estava proporcional à população, no entanto, a quantidade de ambulâncias de SAV estava insuficiente para o adequado atendimento. Em função da alta demanda atendida pela instituição, a existência somente dessas três modalidades de veículos e a quantidade reduzida de ambulâncias de SAV limitam a assistência prestada.

Minayo e Deslandes ${ }^{14}$ pontuam que Brasília apresenta em seu sistema préhospitalar apenas as ambulâncias do tipo B e D, assemelhando-se ao SAMU Fortaleza. Ressaltam que isto indica uma reduzida diversidade de opções para atender às necessidades desse vasto complexo urbano.

A articulação e a integração com outros serviços denotam a qualidade do atendimento pré-hospitalar, devendo ocorrer não somente entre as categorias profissionais que compõem a equipe de atendimento. A assistência nesse nível de atenção necessita de profissionais de outros setores para que o trabalho seja efetivo e realizado por meio de relações horizontais de interdisciplinaridade, com suporte na troca de saberes que se constituem no campo da saúde coletiva ${ }^{17}$. $\mathrm{O}$ trabalho articulado é requisito à assistência integral às vítimas que necessitam de abordagem integrada, devendo existir troca e cooperação dos profissionais em prol da pessoa assistida.

No que se refere ao vínculo empregatício, pesquisa acerca da caracterização gerencial dos hospitais filantrópicos no Brasil revela resultados que vão ao encontro dos achados deste estudo, em que os profissionais de nível superior com vínculo 
empregatício terceirizado prevalecem na organização hospitalar estudada ${ }^{18}$. A terceirização é realizada não para o aumento da eficiência e da produtividade, mas para reduzir custos por meio da exploração de relações precárias do trabalho. $\mathrm{O}$ aumento deste tipo de contrato contribui para a precarização do processo de trabalho, submetendo a risco a coesão dos coletivos. Assim, inserem-se nas relações de trabalho elementos que desestabilizam a confiabilidade no desenvolvimento e na própria produção final do trabalho ${ }^{19,20}$.

Transferindo as afirmações desses estudiosos para a realidade do SAMU, a dominância de vínculos frágeis entre os profissionais de nível superior e a instituição pode ensejar insatisfações, desinteresse ou mesmo desresponsabilização em relação produto final da prática cotidiana de salvar vidas A instabilidade dos vínculos vai de encontro aos pré-requisitos e compromissos para a captação de recursos federais para a implantação do pré-hospitalar móvel. Nesse sentido, a Portaria $\mathrm{n}^{\circ} 1.864$ /GM exige que seja comprovada a contratação de recursos humanos, não sendo permitida a precariedade de vínculos nas relações de trabalho ${ }^{15}$.

Em um contexto mais amplo, estes achados podem refletir na precarização do processo de trabalho no SUS, com possibilidade de interferência na motivação da educação permanente. Esta funcionaria como propulsora para despertar no profissional o interesse em conhecer as políticas de saúde e o Sistema Único de Saúde como um todo, possibilitando uma prática integrada e contextualizada com os processos políticos.

Insere-se nesse espaço de debate a capacitação específica para o APH em que, de acordo com a PNAU, a capacitação das equipes de saúde deve ocorrer sob um enfoque estratégico promocional, abrangendo a gestão e a atenção, envolvendo profissionais de nível superior e técnico, em consonância com as diretrizes do SUS e embasada nos pólos de educação permanente ${ }^{4}$.

No âmbito da preparação do profissional para a atuação no serviço de atendimento pré-hospitalar, a atualização é tão impor- tante quanto a capacitação específica para melhorar a qualidade da assistência. Estudo acerca da implementação do APH demonstrou que há adequação entre os recursos humanos e a função desempenhada pelos profissionais no serviço. Em Manaus, Recife, Rio de Janeiro e Curitiba, as equipes foram capacitadas com o Advanced Trauma Life Support (ATLS). O gestor de Brasília, porém, enfatizou que as equipes possuem deficiências no que concerne a capacitação ${ }^{14}$.

Aprofundando essa reflexão, ressalta-se que os gestores dos serviços pré-hospitalares devem ser corresponsáveis pela capacitação e atualização de seus profissionais. A oferta de cursos pelo serviço deve ser uma constante no cotidiano do trabalho, bem como o estímulo para a busca da ampliação do conhecimento. Além da oferta de atividades, deve ser uma preocupação desses gestores a criação de possibilidades para que o profissional participe dos cursos oferecidos, conciliando a oferta com a jornada de trabalho, o que se estabelece como um desafio para a gestão.

Estudo sobre a educação em serviço para profissionais de saúde do SAMU de Porto Alegre relata a dificuldade em conciliar a oferta de cursos e treinamentos com as escalas verticais de trabalho. Apesar das tentativas de organização das aulas em diferentes horários, o que permitiria a escolha por preferência e disponibilidade fora dos horários habituais de trabalho, a adesão dos profissionais às atividades foi baixa ${ }^{21}$.

Corrobora-se a noção de que a gestão deve reconhecer que a amplitude das ações necessárias ao serviço vão além da prestação da assistência. O compromisso com a educação permanente não deve ser atribuído somente ao trabalhador. Ao administrar a instituição, o gestor deve priorizar a qualificação do profissional. Do contrário, as ações voltadas para a capacitação e atualização destes não podem ser consideradas como educação permanente, uma vez que não estão inseridas na rotina do serviço, mas sim como atividades contingentes, dispersas e desmembradas da organização do trabalho ${ }^{21}$. 
Uma alternativa para reduzir a escassez de recursos humanos qualificados seria considerar a educação permanente como carga horária trabalhada na rotina do serviço, favorecendo a participação dos profissionais. Nas seções de educação permanente (cursos, palestras, treinamentos, simulados), poderiam ser instituídos protocolos de atendimento como produto final de cada atividade. Assim, a educação permanente, além de se estabelecer como meio de qualificação profissional, seria uma ferramenta para a sistematização da assistência prestada.

Quanto ao tempo de resposta total, que constitui um dos indicadores de desempenho e qualidade do serviço pré-hospitalar, a comparação entre os estudos que abordaram esta variável pode ser comprometida pelas diferenças metodológicas de sua aferição. Diante desta limitação, expõem-se aqui alguns resultados de pesquisas acerca do tempo de resposta.

O tempo médio entre a recepção do chamado e a entrada do paciente no hospital foi de 35,6 minutos em Montreal (Canadá). Na Irlanda do Norte, este foi aferido em 30,5 minutos, o que se aproximou dos resultados deste estudo ${ }^{22,23}$. No Brasil, o tempo médio de resposta total mensurado foi de 41,0 minutos em estudo sobre a sobrevivência após acidentes de trânsito, no Município de São Paulo ${ }^{24}$. Considerando que a presteza do atendimento influencia intensamente na sobrevivência das vítimas atendidas pelo APH, o tempo gasto entre o acionamento do serviço e o estabalecimento do tratamento definitivo deve ser o menor possível, a fim de garantir o melhor prognóstico.

O Comittee on Trauma of American College of Surgeons ${ }^{25}$ estabelece que o intervalo máximo para a instituição dos primeiros procedimentos em casos graves é de 20 minutos, pelo fato de que nas primeiras horas após o trauma ocorre o maior índice de mortalidade. De modo geral, mais de $50 \%$ das vítimas de trauma não resistem as primeiras 24 horas. Vários fatores exercem influência sobre o tempo de resposta total. Estudiosos evidenciam que entre eles estão a distância do local da ocorrência, as condições impostas pelo trânsito, a disponibilidade de ambulâncias e a articulação com o pré-hospitalar fixo e hospitais da rede de atendimento $^{14,24}$. O conceito de tempo de resposta total definido corretamente pelos profissionais médicos pode ser atribuído ao fato de que esses profissionais participam mais diretamente das atividades da gestão o que, possivelmente, lhes confere o contato maior com as políticas de saúde, entre elas a que institui o componente pré-hospitalar móvel.

Os problemas no sistema de referência e contrarreferência comprometem diretamente a continuidade do cuidado ao usuário do SUS. As lacunas na integração entre o pré-hospitalar e a rede hospitalar são agravadas pela falta de vagas, deficiência que atinge várias capitais brasileiras ${ }^{14}$. Salienta-se que é rotina no cotidiano do APH o fato de os pacientes ficarem nos hospitais ocupando as macas das ambulâncias à espera de um leito. Nesse ínterim, o veículo de urgência e a equipe ficam presos no hospital, ocasionando transtornos e prejudicando o desenvolvimento do atendimento pré-hospitalar.

O retardamento da recepção do paciente no nível terciário de atenção fere dois princípios doutrinários do Sistema Único de Saúde: a universalidade e a integralidade. Prosseguindo no contexto amplo das políticas públicas, ressalta-se que os profissionais das equipes de atendimento do SAMU necessitam ter acesso ao documento oficial da Política Nacional de Atenção às Urgências, para que possam, no cotidiano do trabalho, reconhecer lacunas e apontar soluções para os problemas identificados. Este acesso possibilitaria ampliar o foco da educação permanente para o campo das políticas de saúde, o que implicaria a consolidação do APH como um dos componentes do SUS.

No contexto do APH, a função das rotinas e protocolos voltados ao atendimento às vítimas do trânsito é sistematizar o atendimento, facilitando a instituição dos cuidados pré-hospitalares. Em cada insti- 
tuição, o desenvolvimento de protocolos assistenciais possui o objetivo de padronizar e sistematizar as condutas, no intuito de reduzir a variação indesejada da prática e melhorar o atendimento ao paciente. É importante ressalvar o fato de que as rotinas e protocolos de um serviço específico correspondem à realidade vivenciada pelos profissionais que nele atuam, devendo ser coletivos, e seu produto final há de ser compartilhado, para que todos tenham uma conduta padronizada ${ }^{26}$.

Diante da análise, os desafios na instituição do SAMU são diversos. Apesar de todas as dificuldades, há que se reconhecer a importância do serviço para a sociedade. Ele veio para padronizar e regular um tipo específico de atendimento fundamental para salvar vidas, com eficácia comprovada por estudos realizados no mundo. Em um contexto mais amplo, é necessário que os gestores do Sistema Único de Saúde assegurem a continuidade da sua implantação e promovam o seu aperfeiçoamento e constante avaliação, a fim de ajustar o seu funcionamento. É preciso garantir a integração do SAMU ao sistema de urgência e emergência propiciando a consolidação do serviço e, consequentemente, das políticas de saúde.

\section{Conclusão}

Ao analisar atendimento pré-hospitalar móvel no Município de Fortaleza de acordo com os profissionais envolvidos, desvelouse a realidade de um serviço em fase de implantação com problemas relativos ao planejamento e de cunho estrutural.

Aqueles relacionados ao planejamento se traduziram como escassez de recursos humanos qualificados, carência de materiais e equipamentos e fragilidade de vínculos e relações de trabalho. A integração limitada entre o SAMU e outros serviços, a falta de vagas nos hospitais e os entraves do sistema referência e contrarreferência conformam-se como problemas estruturais do Sistema Único de Saúde.

Ao mesmo tempo em que descreve o atendimento pré-hospitalar sob a ótica dos profissionais, os resultados desta pesquisa sinalizam que a busca de soluções para os problemas detectados requer novas visões e perspectivas que privilegiem a consolidação do serviço e das políticas de saúde.

\section{Referências}

1. Ministério da Saúde. Portaria GM n².048, de 05 de novembro de 2002. Política Nacional de Atenção às Urgências. Brasília: Ministério da Saúde; 2006.

2. Cabral APS, Souza WV. Serviço de Atendimento Móvel de Urgência (SAMU): análise da demanda e sua distribuição espacial em uma cidade do Nordeste brasileiro. Rev Bras Epidemiol 2008; 11: 530-40.

3. Vieira CMS, Mussi FC. A Implantação do Projeto de Atendimento Móvel de Urgência em Salvador/BA: panorama e desafios. Rev Esc Enferm USP 2008; 42: 7937.

4. Ministério da Saúde. Política Nacional de Atenção às Urgências. Brasília: Ministério da Saúde; 2006.

5. Ministério da Saúde. Regulação Médica das Urgências. Brasília: Ministério da Saúde; 2006

6. Malvestio MAA, Sousa RMC. Suporte avançado à vida: atendimento a vítimas de acidentes de trânsito. Rev Saúde Pública 2002; 36: 584-9.
7. Ministério da Saúde. Política Nacional de Redução da Morbimortalidade por Acidentes e Violências. Brasília: Ministério da Saúde; 2001.

8. Organização Panamericana de Saúde. Organização Mundial de Saúde. Respostas do Setor Saúde ao Desafio para um Trânsito mais Seguro nas Américas. Informe sobre a Conferência Panamericana sobre Segurança no Trânsito. Brasília; 2005.

9. Samu. Serviço de Atendimento Móvel de Urgência- 192. Etapas, Localidades e Cidades Atendidas. Disponível em http://portal.saude.gov.br/portal/saúde/visualizar_ texto.cfm?idtxt $=23745 \&$ janela $=1$. [Acessado em 25 de junho de 2009]

10. Ministério da Saúde. Conselho Nacional de Ética em Pesquisa. Resolução 196/96. Disponível em http:// conselho.saude.gov.br/comissao/conep/resolucao.html. [Acessado em 03 de maio de 2007]

11. Zapparoli AS, Marziale MHP. Risco ocupacional em unidades de Suporte Básico e Avançado de Vida em emergências. Rev Bras Enferm 2006; 59: 41-6. 
12. Lopes ACS, Oliveira AC, Silva JT. Paiva MHRS. Adesão à precauções padrão pela equipe do atendimento pré-hospitalar móvel de Belo Horizonte, Minas Gerais, Brasil. Cad Saúde Pública 2008; 24: 1387-96.

13. Mesquita Filho M, Mello Jorge MH. Características da morbidade por causas externas em serviço de urgência. Rev Bras Epidemiol 2007; 10: 679-91.

14. Minayo MCS, Deslandes SF. Análise da implantação do sistema de atendimento pré-hospitalar móvel em cinco capitais brasileiras. Cad Saúde Pública 2008; 24: 1877-86.

15. Ministério da Saúde. Portaria GM nº 1.864, de 29 de setembro de 2003. Institui o componente pré-hospitalar móvel da Política Nacional de Atenção à Urgência, por intermédio da implantação de Serviços de Atendimento Móvel de Urgência em municípios e regiões em todo o território brasileiro: SAMU 192. Política Nacional de Atenção às Urgências. Brasília: Ministério da Saúde; 2006.

16. Instituto Brasileiro de Geografia e Estatística. Contagem da População 2007. Disponível em http:/ / www.ibge. gov.br/home/estatistica/populacao/contagem2007/ popmunic2007layoutTCU14112007.pdf. [Acessado em 21 de novembro de 2008]

17. Alves M, Ramos FRS, Penna CCM. O trabalho interdisciplinar: aproximações possíveis na visão de enfermeiras de uma unidade de emergência. Texto Contexto - Enferm 2005; 14: 323-31.

18. Lima SML, Barbosa PR, Portela MC, Ugá MAD, Vasconcellos MM, Gerschman S. Caracterização gerencial dos hospitais filantrópicos no Brasil. Cad Saúde Pública 2004; 20: 1249-61.

19. Chillida MSP, Cocco MIM. Saúde do trabalhador e terceirização: perfil de trabalhadores de serviço de limpeza hospitalar. Rev Latin-Am Enfermagem 2004; 12: 271-6.
20. Figueiredo M, Alvarez D, Athayde M, Suarez JD, Pereira R. Reestruturação produtiva, terceirização e relações de trabalho na indústria petrolífera offshore da Bacia de Campos (RJ). Gest Prod 2007; 14: 55-68.

21. Ciconet RM, Marques GQ, Lima MAD. Educação em serviço para profissionais de saúde do Serviço de Atendimento Móvel de Urgência (SAMU): relato de experiência de Porto Alegre - RS. Interface 2008; 12: 65966.

22. Sampalis JS, Lavoie A, Willians JI, Mulder DS, Kalina M. Impact of on-site care, prehospital time, and level of in-hospital care in survival in severely injured patients. $J$ Trauma 1993; 34: 252-61.

23. McNichol BP. The golden hour and prehospital trauma care. Injury 1994; 25: 251-4.

24. Ladeira RM, Barreto SM. Fatores associados ao uso de serviço de atenção pré-hospitalar por vítimas de acidentes de trânsito. Cad Saúde Pública 2008; 24: 28794 .

25. Committee on Trauma of the American College of Surgeons. Hospital and prehospital resources for optimal care of the injured patient. Bull Am Coll Surg 1986; 71: 4-23.

26. Rocha PM, Fernandes AK, Nogueira F, Piovesan DM, Kang S, Franciscatto E, Millant T, Hoffmann C, Polanczyk CA, Barreto SSM, Dalcin PTR. Efeito da implantação de um protocolo assistencial de asma aguda no serviço de emergência de um hospital universitário. J Bras Pneum 2004; 30: 94-101.

Recebido em: 12/03/09 Versão final reapresentada em: 26/06/09 Aprovado em: 28/09/09 